This item was submitted to Loughborough's Research Repository by the author.

Items in Figshare are protected by copyright, with all rights reserved, unless otherwise indicated.

\title{
Predictors of paternal and maternal controlling feeding practices with 2- to 5- year-old children
}

PLEASE CITE THE PUBLISHED VERSION

http://dx.doi.org/10.1016/j.jneb.2010.03.001

PUBLISHER

Elsevier (c) Society for Nutrition Education and Behavior

VERSION

SMUR (Submitted Manuscript Under Review)

LICENCE

CC BY-NC-ND 4.0

REPOSITORY RECORD

Haycraft, Emma, and Jacqueline Blissett. 2019. "Predictors of Paternal and Maternal Controlling Feeding Practices with 2- to 5-year-old Children”. figshare. https://hdl.handle.net/2134/11243. 
This item was submitted to Loughborough's Institutional Repository (https://dspace.lboro.ac.uk/) by the author and is made available under the following Creative Commons Licence conditions.

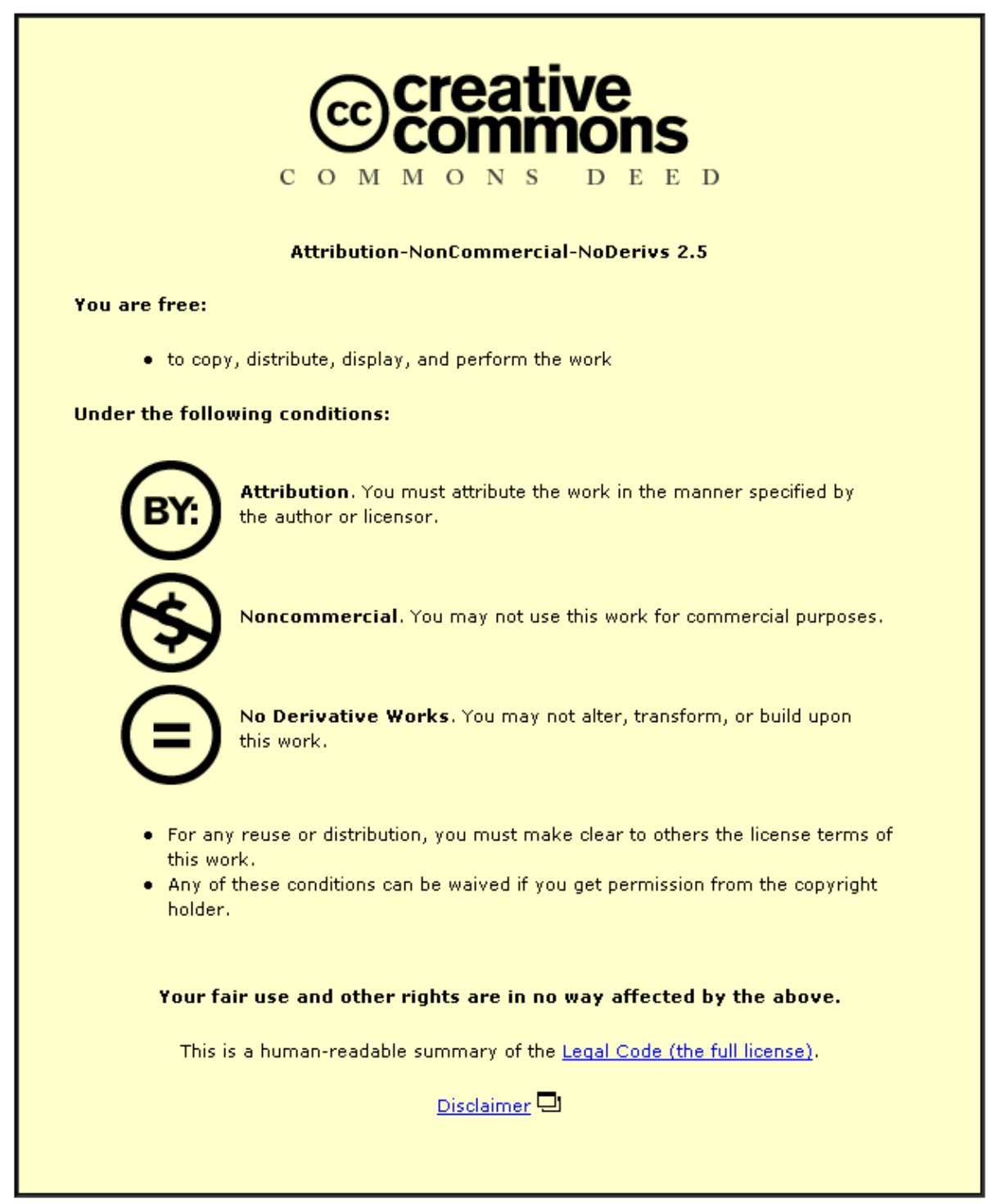

For the full text of this licence, please go to: http://creativecommons.org/licenses/by-nc-nd/2.5/ 
Please note: This is a Research Article, intended for a Regular Issue of JNEB.

\section{Running head: PREDICTORS OF FEEDING PRACTICES}

\section{PREDICTORS OF PATERNAL AND MATERNAL CONTROLLING FEEDING}

PRACTICES WITH 2 TO 5-YEAR-OLD CHILDREN

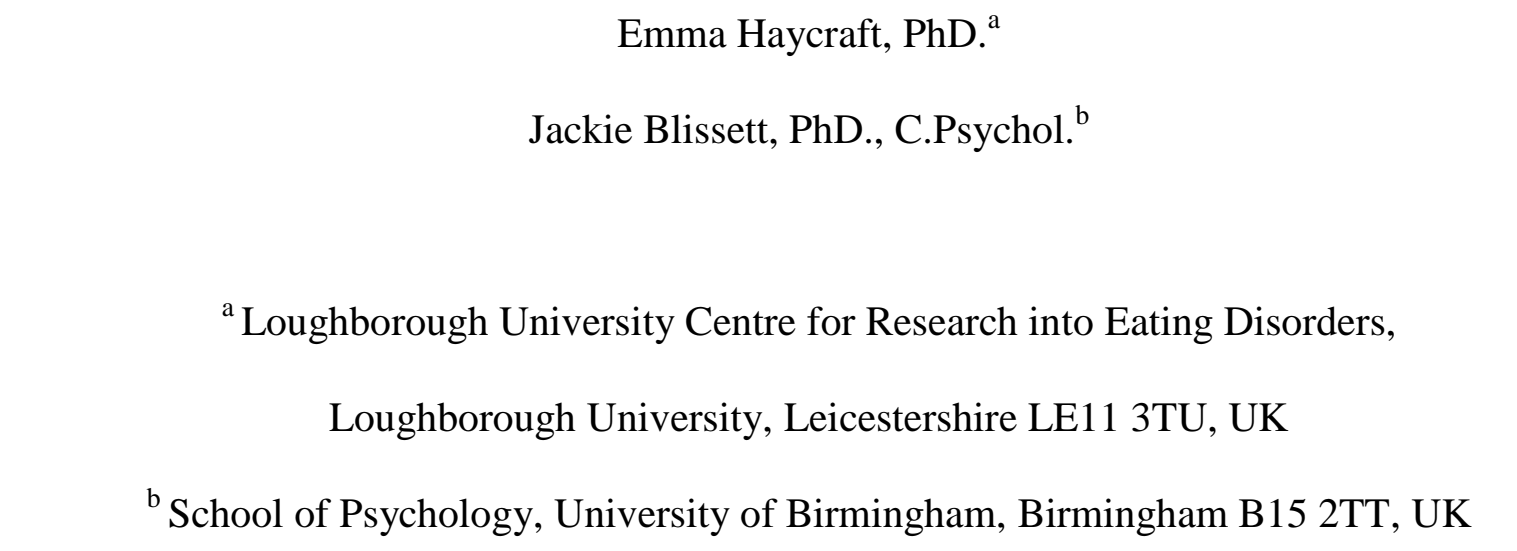
Research into Eating Disorders, School of Sport, Exercise and Health Sciences, Loughborough University, Leicestershire LE11 3TU, UK.

Email: E.Haycraft@lboro.ac.uk; [Not for publication] Tel. +44 (0)1509 228160; Fax. +44 (0)1509 223940 


\section{ABSTRACT}

30 Objective: This study aimed to identify predictors of controlling feeding practices in both mothers and fathers of young children.

Design: Cross-sectional, questionnaire design.

Setting: Nursery schools within the United Kingdom recruited participants.

Participants: Ninety-six mothers and fathers, comprising 48 mother-father pairs of male and female children aged 2 to 5 years.

Main outcome measures: Parents' child feeding practices, eating psychopathology, general mental health symptomology, and their children's eating behaviors and temperament.

Analysis: Preliminary correlations; stepwise regressions.

Results: Maternal controlling feeding was predicted by children's eating behaviors (emotional over- and under-eating), child temperament (sociability), and maternal general mental health symptoms. Paternal reports of children's eating behaviors (slow eating and emotional under-eating) were the only significant predictors of fathers' controlling feeding practices.

Conclusions and implications: Mothers' and fathers' feeding practices seem to be better

linked to child characteristics than to the presence of eating psychopathology symptoms.

Children's emotional eating predicted all three controlling feeding practices in mothers and warrants further study to elucidate the causal nature of this relationship.

[Abstract word count $=165]$

51 Key words: child feeding; eating; temperament; pressure to eat; restriction. 


\section{PREDICTORS OF PATERNAL AND MATERNAL CONTROLLING FEEDING}

\section{PRACTICES WITH 2 TO 5-YEAR-OLD CHILDREN}

Over-controlling child feeding practices may contribute to the development of childhood obesity, particularly in white, middle class samples $(1,2)$. Practices such as excessive overt restriction of children's food intake may promote childhood overweight by inhibiting the child's development of self-regulatory processes in appetite regulation $(3,1)$.

Researchers must endeavour to discover what circumstances might lead parents to use these feeding practices with the aim of reducing practices which may unintentionally promote childhood obesity.

Recent research has found children's temperament, feeding behaviors, and maternal mental health to predict mothers' controlling feeding practices with their 1 and 2-year-old children (4). In addition, the use of controlling feeding practices is thought to be fairly stable $(4,5)$. What is not known, however, is whether these same factors are predictive of the controlling feeding practices used by mothers of children aged 2 to 5 years, and whether fathers' feeding practices have similar predictors.

Symptoms of eating psychopathology in parents have been commonly associated with the use of overly controlling feeding practices in both non-clinical and clinical samples. For example, mothers with eating psychopathology or with their own eating and weight issues have been found to be less sensitive and more coercive during mealtime interactions with their children (6), use more restrictive child feeding practices with their daughters (7), and carry out high levels of monitoring of their daughters' food intake (8). Fathers' dieting attitudes and behaviors have also been shown to be important in predicting their sons' and daughters' later weight concerns and dieting behaviors (9).

Other types of parental psychopathology have also been related to controlling feeding interactions. Symptoms of anxiety and obsessive-compulsive disorder (OCD) have both been 
related to greater maternal restriction of children's food intake $(7,10)$. Depression has been associated with increased application of pressure to eat in mothers of 5-year-old girls (7); a practice which can also impair children's recognition of their internal responses to hunger and satiety (3). Although studies examining fathers' psychopathology and feeding interactions are scarce, a range of mental health symptoms have been shown to relate to fathers' use of more pressuring and restrictive feeding practices with their young children (11).

While considerable effort has been devoted to identifying the parental factors that predict maladaptive feeding practices, it is likely that there is an interaction between child characteristics and parental practices. Much of the literature implies a causal relationship between feeding practices and children's subsequent eating behavior and weight gain, but parents are also likely to moderate their feeding practices according to their children's eating behavior. For example, previous research (4) has found children's eating behaviors to be important predictors of maternal controlling feeding practices with children at both one and two years of age. It is likely that there is a multidirectional interaction between children's eating behavior, weight status and parents' feeding behaviors. It has been suggested that factors such as fussiness can be problematic for parents to manage (see 12) and children's picky eating has been associated with parents applying pressure on their children to eat (13). Hence, children's eating behaviors are expected to be good predictors of mothers' and fathers' feeding practices with their 2 to 5-year-old children.

Difficult infant temperament can be related to problematic feeding interactions between mother and child (14), and to children's unwillingness to try novel foods (15). The role of infant temperament in non-clinical feeding difficulties has been widely established, while its role in determining parents' feeding practices is less well understood. Infant temperament, specifically maternal perceptions of infant dullness and unpredictability, has previously been found to predict maternal control with 1- and 2-year-old infants (4), but no work to date has examined temperament as a predictor of controlling feeding practices in a 
105

sample of older children, or as a potential predictor of fathers' feeding practices.

The majority of research in the child feeding domain has considered mothers, yet research suggests that fathers play important roles in child feeding (16-18). It is widely accepted that mothers tend to have more responsibility than fathers for feeding a child and for deciding on the types of food that the child eats. This difference has been reflected in the literature with studies finding that mothers report having significantly greater feeding responsibility than fathers, even in dual income families (16). Hence the current study aims to further examine differences between mothers and fathers; this time examining which of a series of factors might best predict mothers' and fathers' controlling feeding practices.

In summary, this study will examine whether measures of children's eating behaviors, children's temperament, and parents' psychopathology will contribute to the explanation of monitoring, pressure to eat and restrictive child feeding practices, in both mothers and fathers of preschool children. Children's less adaptive eating behaviors (e.g., slowness, emotional eating, and fussiness), difficult child temperament (e.g., less sociable, more emotional), and greater reported levels of parental psychopathology were all hypothesised to predict more controlling maternal and paternal feeding practices. In this exploratory, cross-sectional study, reference to 'prediction' and 'predictors' refers to statistical prediction and does not imply assumptions about causal relationships. Furthermore, differences in the predictors contained within final models for mothers and for fathers were expected, in light of factors such as mother-father differences in child feeding responsibility.

\section{Participants}

\section{METHOD}

Ninety-six parents constituting 48 mother-father pairs of children aged between 2 to 5 years took part in this study. The participants were recruited with the assistance of private and Local Education Authority Nursery Schools from the West Midlands and 
131 Cambridgeshire, UK. Approximately 2000 questionnaires were sent to nurseries and,

132 presuming that all of these were distributed, this suggests a response rate of $9.4 \%$. Pairs of

133 returned questionnaires were matched up using an identifier code and participants were

134 excluded if they were not part of a pair of returned questionnaires or if they were not

135 cohabiting with their partner $(n=72)$. Respondents were also excluded if the child they

136 reported on was under 24 or over 60 months of age ( $n=10$ pairs). The mean age of the

137 mothers was 35 years ( $S D 4.28$, range 23 to 46 years) and the mean age of the fathers was 37

138 years (SD 5.00, range 26 to 49 years). The mean age of the children was 42 months (SD 9.00,

139 range 24 to 59 months). Eighty percent of mothers and $83 \%$ of fathers had

140 managerial/professional occupations, as assessed by the National Statistics Socio-Economic

141 Classification self-coded method (NSSEC; 19). The NSSEC comprises four questions which

142 ask: 1. Whether the individual is (or was, when last in employment) an employer, self-

143 employed or an employee; 2. The size of organisation in which they work(ed); 3. Supervisory

144 status; and, 4. Current or most recent occupation. Responses to these questions lead to one of

145 the following five classifications: managerial/professional occupations; intermediate

146 occupations; small employers and own account workers; lower supervisory and technical

147 occupations; semi-routine and routine occupations. Ethnicity data were not collected, but the

148 nurseries involved in this study served primarily white neighbourhoods. Seventy-nine percent

$149(n=38)$ of the parents were married and $21 \%(n=10)$ were cohabiting.

Measures and procedure

Following institutional review board ethical approval, informed consent was obtained

153 from mothers and fathers and the following self-report questionnaires were administered via

154 nurseries. Questionnaires were returned via post to the researchers.

155 Child Feeding Questionnaire (CFQ) (20): The CFQ is a valid, reliable measure (20)

156 which assesses parental beliefs, attitudes and practices regarding child feeding, and examines 
157 parental concerns about childhood obesity. The measure is suitable for use by mothers and

158 fathers. Parents reported on the following feeding practices: Monitoring (keeping track of

159 children's unhealthy food intake); Pressure to Eat (encouragement to eat more food); and

160 Restriction (limiting consumption of certain foods; using food as rewards). Responses are

161 indicated using a 5-point scale with response options ranging from "never" to "always" for

162 monitoring and from "disagree" to "agree" for the pressure to eat and restriction subscales.

163 Mean scores are calculated for each subscale with possible mean scores ranging from 1 to 5.

164 Higher scores indicate greater reports of each feeding practice.

165 Child Eating Behaviour Questionnaire (CEBQ) (12): The CEBQ assesses children's

166 eating styles based on parental reports of their child's behavior and has been shown to be

167 valid and reliable (12). Seven subscales which examine children's positive and negative

168 reactions to food were used: Food Responsiveness; Enjoyment of Food; Satiety

169 Responsiveness; Food Fussiness; Slowness in Eating; Emotional Over-Eating; and Emotional

170 Under-Eating. The Desire to Drink subscale was excluded from this study. The questions are

171 rated on a 5-point scale (1=never to 5=always), and five items are reverse scored. Mean

172 scores ranging from 1 to 5 are calculated for each subscale and higher scores indicate a

173 greater prevalence of that particular eating behavior.

174 EAS Temperament Survey for Children: Parental Ratings (EAS) (21): The EAS is a

175 parental self-report measure of children's temperament, comprising four subscales:

176 Emotionality; Activity; Sociability; and Shyness. Twenty statements are responded to on a 5-

177 point scale, ranging from 1 (not characteristic of your child) to 5 (very characteristic of your

178 child). Six items are reverse-scored. Mean scores from 1 to 5 are calculated for each

179 subscale. The EAS has previously demonstrated adequate psychometric properties (22).

180 Eating Disorder Inventory-2 (EDI-2) (23): The EDI-2 is a self-report measure of

181 eating disorder symptoms. It has been validated for use with non-clinical samples of men as

182 well as women (24) and has been found to display good reliability and validity (23). Only 
183 questions pertaining to the three eating subscales were administered in this study: Drive for

184 Thinness; Bulimia; and Body Dissatisfaction. Each item is scored on a 6-point scale, with

185 options ranging from "always" to "never". Six items are reverse-scored. A total EDI score

186 was calculated by summing the responses to all 23 questions. Possible scores ranged from 0

187 to 69 . Higher scores indicate greater levels of pathology.

188 Brief Symptom Inventory (BSI) (25): The BSI is a measure of current, point-in-time,

189 psychological symptom status which is widely used and has good levels of reliability and

190 validity (26). The BSI consists of 53 questions and response options range from 0 (not at all)

191 to 4 (extremely). Higher scores indicate a greater experience of the symptom. As per

192 recommendations in the BSI manual (25), raw scores were converted to T-scores with

193 possible T-scores ranging from 33 to 80 . For the purposes of this study, only the overall

194 measure of current level of symptomology (the Global Severity Index) will be used. The

195 Global Severity Index (GSI) is the most sensitive single indicator of the respondent's distress,

196 and combines the number of symptoms reported with information about intensity of distress

197 (25).

198 Additional information: Parents were asked to report height and weight data for their

199 children. In addition, researchers completed height and weight measurements for $33 \%(n=16)$

200 of the children in the current sample who were visited at home. Pearson's one-tailed

201 correlations showed excellent concordance between parents' reports of their children's

202 heights and weights and those obtained by the researcher, suggesting reliability in these data

203 ( $r$ values .791 to .856 , p values .001 to $<.001$ ). Children's Body Mass Index (BMI) scores

204 were calculated and then converted into BMI standard deviation scores (BMI SDS) using the

205 Child Growth Foundation Reference Curves Disc (27), which standardises BMI for child age

206 and gender. The mean child BMI SDS was 0.02 (SD 1.73, range -3.46 to 3.04). Using the

207 international definitions for overweight and obesity in children aged over 2-years-old

208 established by Cole, Bellizzi, Flegal and Dietz (28), four children in this sample (8\%) were at 
risk of overweight in adulthood (i.e. having a $\mathrm{BMI} \geq 25$ at age $18+$ ) and five children (10\%) were classified as at later risk of obesity (i.e. a $\mathrm{BMI} \geq 30$ in adulthood). Information was requested about breastfeeding duration and, using the NSSEC, about mothers' and fathers' socio-economic status (SES) (19), in addition to child BMI data, because these factors have previously demonstrated relationships with eating, child BMI and controlling feeding practices $(4,20,29,30)$. Mothers and fathers were also asked to indicate the number of meals, during a typical week, that they ate with their child.

\section{Data analysis}

The majority of the data were identified as being non-normally distributed and so nonparametric tests were used where possible. Significance levels were set at $p<.05$ for all analyses.

There were no significant differences in parents' feeding practices dependent on the gender of the child. Therefore, child gender was not examined further in this study.

Two-tailed Spearman's correlations were conducted to examine the relationships between potentially confounding variables (child BMI SDS, child and parent age, parents' SES, and breastfeeding duration) with mothers' and fathers' child feeding practices. There were no significant correlations between any of these factors with maternal or paternal reported feeding practices and so these factors were not entered into any of the subsequent regression analyses. It is particularly noteworthy that child BMI SDS was not related to either maternal or paternal child feeding practices and so was not controlled for in any of the following regressions.

As the sample size was modest, and in order to maintain power when using regressions, a series of two-tailed Spearman's correlations were initially conducted to identify significant correlates for entry into the regression equations. 
A series of stepwise multiple linear regressions were then run to test the hypothesis that children's maladaptive eating behaviors (e.g., slowness, emotional eating, and fussiness),

236 difficult child temperament, and greater reported parental psychopathology were likely to predict more controlling parental feeding practices. Mothers' reports were used to predict their feeding practices and fathers' feeding practices were predicted from paternal reports, in order to examine how each parent's perceptions of their child are related to their reported 240 child feeding practices. The distribution of the CFQ subscales is noteworthy, being a mixture 241 of normally (maternal reports of pressure to eat and restriction) and non-normally (all paternal 242 CFQ subscales and maternal monitoring) distributed. There is no non-parametric equivalent of regression analysis, but the use of multiple regression analyses was deemed suitable on these data as Field (31) has stated that "predictors do not need to be normally distributed" (p.170), rather it is the normal distribution of the errors which is important. Hence, the data were screened for violations to the regression assumptions as outlined by Tabachnick and Fidell (32). Stepwise regressions were chosen in view of the exploratory nature of these analyses. Six regressions were conducted to examine the predictive value of children's eating behaviors, children's temperament and parent mental health on mothers' and fathers' feeding 250 practices.

\section{RESULTS}

Descriptive statistics

The data obtained from mothers and fathers in the current study (Table 1) are broadly in line with data obtained in other studies which have used the CFQ (16), CEBQ (12), EAS (22), EDI-2 (11) and BSI (25). The reliability of these measures with the current sample 
260 GSI score of 63 or more, which is the cut-off for clinical concern or "caseness" $(25, \mathrm{p} .32)$.

261 Four mothers (8\%) but no fathers had scores on the EDI-2 that indicated potential clinical 262 caseness in their reports of their drive for thinness. This difference in EDI-2 scores is in line 263 with findings from another sample of parents of preschoolers (16), where mothers reported 264 more eating disorder symptoms than fathers. No participants' scores reached a clinically 265 significant level of bulimia symptoms. Mothers reported eating a mean of 15 meals (SD 4.06) 266 per week with their child and for fathers, the mean was 11 meals ( $S D$ 3.51). This is in 267 accordance with previous work which has found both fathers and mothers in two parent 268 families to eat frequently with their children (17). analyses to identify predictors of mothers' and of fathers' feeding practices. It is noteworthy that maternal and paternal reports on the CEBQ were all positively and significantly associated $(\mathrm{p}<.05)$ with the exception of emotional under-eating, for which there was evidence of a trend ( $\mathrm{p}=.075)$ (data not shown), suggesting similarity in parental judgement of children's eating behaviors. predicted by lower levels of children's emotional over-eating and lower child sociability

283 (Table 3). Higher levels of paternal monitoring were predicted by lower levels of children's emotional under-eating. Child temperament did not predict paternal monitoring and neither 
monitoring.

Predictors of maternal and paternal pressure to eat: The significant predictors of higher levels of maternal pressure to eat were mothers' reports of their children emotionally under-eating and higher levels of maternal general mental health symptoms (GSI). Children's temperament and maternal eating disorder symptoms did not predict maternal use of pressure. Children's slowness in eating was the only significant predictor of greater paternal use of pressure to eat. Paternal mental health symptoms and children's temperament did not significantly predict fathers' pressuring feeding practices.

\section{Predictors of maternal and paternal restriction: Maternal restriction was solely} predicted by higher levels of children's emotional over-eating. Children's temperament and maternal mental health did not predict mothers' restrictive feeding practices. For fathers, their use of restriction was predicted by children's slowness in eating. Fathers' own mental health scores and children's temperament were not significant predictors of their use of restriction.

$$
\text { controlling child feeding practices in a sample of mothers and fathers of 2- to 5-year-old }
$$
children. It also aimed to highlight differences between mothers and fathers in the patterns of significant predictors of their feeding practices. Different patterns of predictors were found for mothers and fathers, and child factors were recurrent predictors of parental feeding practices. Fathers' feeding practices were predicted entirely by their children's slow or undereating behaviors. In contrast, maternal feeding practices were predicted by a more complex set of predictors, including children's emotional eating behavior, child sociability and maternal mental health symptoms.

For mothers, monitoring behavior was best predicted by their reports of their child's sociability and emotional over-eating. Having a less sociable child predicted greater 
312 monitoring of children's snack or junk food intake. Previous studies have associated difficult,

313 unsociable child temperaments with negative mealtimes and difficult feeding interactions

$314(14,15)$. Hence, it is possible that increased monitoring is a response to eating difficulties in

315 children with unsociable temperaments. Additionally, mothers tended to monitor their

316 children's food intake more when their children were less likely to emotionally over-eat.

317 Moderate levels of monitoring can be adaptive (2). Therefore, this finding may indicate that

318 these mothers are implementing appropriate covert control over their children's eating, by

319 monitoring their food intake, and that these children have healthier eating attitudes, as

320 illustrated by them being less likely to emotionally over-eat.

Lower reported levels of children's emotional under-eating predicted more paternal

322 monitoring of their children's food intake. These findings also accord with the suggestion

323 that appropriate levels of monitoring can relate to healthier eating behaviors, as fathers who

324 keep track of their children's food consumption have children who are less likely to

325 emotionally under-eat.

326 Mothers' reports of emotional under-eating in their children and symptoms of mental

327 health problems best predicted maternal reports of applying pressure for their children to eat.

328 Pressure to eat often occurs when parents feel that their child is eating insufficient food

329 (7,13). If mothers perceive their children to be under-eating, albeit in response to emotional

330 situations, then it is unsurprising if this predicts them pressuring their children to eat.

331 However, it could also be that it is the mothers' pressuring feeding behaviors which lead to

332 their children refusing food or under-eating, due to the creation of a negative environment

333 surrounding eating and mealtimes. The association between negative mealtimes and food

334 refusal has been found in previous research (33). Maternal mental health symptoms also

335 predicted maternal use of pressure. Mental health symptoms have previously been related to

336 the use of pressuring $(7,11)$ and restrictive $(7,10)$ feeding practices. It is suggested that these

337 symptoms may impair parents' sensitivity and responsiveness to their children, which may 
extend into the feeding domain, with feeding interactions being more controlling. It is noteworthy that this finding accords with that of a previous study (4), where mental health symptoms also predicted pressure to eat in a sample of mothers with 2-year-old children. Children's slowness in eating predicted fathers' application of pressure for their children to eat. Children who are slow eaters may elicit parental pressure to finish the meal, or alternatively, paternal pressure may create a negative mealtime, leading to children eating more slowly. Previous literature has suggested that children's slow eating can be an indicator of heightened responsiveness to satiety (34). Pressuring children who parents perceive are slow eaters may suggest that these parents are encouraging their children to eat beyond satiety.

Children's emotional over-eating was the only predictor of restrictive feeding practices in mothers. Mothers who use restrictive feeding practices may be particularly sensitive to their children's eating behavior in response to emotion. If mothers feel that their children tend to eat more in response to emotional situations, then they may restrict their children's unhealthy food intake as a way of ensuring that their child eats more healthily. It is also possible that maternal tendencies to impose restriction may be teaching children emotional responses to food, rather than allowing children autonomy in their food choices. Paternal restriction was predicted by children's slowness in eating. Children may eat slowly as a response to higher levels of paternal restriction; mealtimes may be more controlling, less enjoyable, constituted of less palatable or preferred foods, potentially leading to greater conflict and to children eating more slowly. Alternatively, it is possible that fathers who perceive their children to be slow eaters are more likely to impose restrictive feeding practices upon their children. Slow eating is often associated with smaller appetites (34), and fathers may perceive that slow eating during meals may be due to snacks that they have eaten prior to the mealtime. Greater restriction of snack and junk foods may thus follow. 
364 findings (4). This study has shown that similar factors to those found with mothers of 1 - and

365 2-year-old children are predictive of both parents' controlling feeding practices with a group

366 of preschool children. Furthermore, this study has shown that these parental feeding practices

367 were frequently predicted by children's eating behaviors. As expected, the factors predicting

368 maternal and paternal use of controlling feeding practices differed, with children's emotional

369 eating significantly predicting mothers' feeding practices and children's slow eating being a

370 recurrent predictor of fathers' feeding practices. There is a tentative suggestion from these

371 data that fathers' feeding practices may be predicted by less complex factors than mothers'

372 feeding practices, with fathers' practices predicted by children's eating behaviors and

373 mothers' practices predicted by their own mental health, their child's emotional eating

374 behaviors, and their child's temperament. While we have demonstrated that practices

375 performed by mothers and fathers are associated with different predictors or correlates, it is

376 important to note that we do not believe that a particular practice performed by a father is

377 necessarily different from the same practice performed by a mother, or that the potential

378 impact of a practice delivered by a mother rather than a father will be necessarily different.

379 Paternal practices may be more clearly, and perhaps more simply, related to the child's eating

380 behaviors, whereas maternal practices may be related to a broader spectrum of factors related

381 to both child and maternal characteristics. These characteristics may not be limited to eating

382 but are perhaps linked to mothers' wider relationships, interactions or mental states. It is

383 possible that each parent's parenting style or emotional tone might moderate the relationship

384 between practice and outcome. For example, paternal pressure to eat may be delivered with a

385 different emotional context than maternal pressure to eat, which in turn may have different

386 effects on children's eating. This remains speculative because our self-report study was

387 unable to examine such factors and as yet no studies have examined these potential

388 differences. 
390

line with some previous findings (35). It is possible that, as most of the children in the current sample were neither overweight nor obese, these parents' feeding practices may be more responsive to their children's eating behaviors and temperament than their child's BMI. It would be interesting to see if the same findings occurred between child BMI SDS and feeding practices in parents of obese or overweight children.

The current study failed to find evidence of eating disorder symptoms as significant predictors of mothers' or fathers' use of controlling feeding practices with their young children. This is contrary to other findings (11) but may be because of the low prevalence of eating psychopathology in this sample, so should not be interpreted as evidence that parental eating psychopathology does not relate to maladaptive feeding practices in clinical groups.

The fact that completed questionnaires were required from cohabiting pairs of mothers and fathers in order to be included in this study, and the fairly high levels of SES and years of education after the age of 16 , limits the generalisability of this study's findings to middle class, well-educated parents residing in the same household. Despite this fact, this study is the first to examine predictors of maternal and paternal feeding practices, and research with fathers within the child feeding domain is sparse and has been called for in previous literature $(16,18)$. The non-clinical sample limits the clinical implications of our findings but further work with clinical samples might find psychopathologies to be stronger predictors of feeding practices in parents with clinically significant psychopathology. A strength of this study is the inclusion of cohabiting mother-father pairs, but a by-product of this is the study's relatively small sample size. A lack of prior research with a similar sample and the same measures prevented us from carrying out a prospective power calculation. However, retrospective power analyses suggest that the effect sizes in our (relatively small) sample range from large (predicting maternal pressure) to small (predicting maternal restriction). Although the self-report nature of this study is not ideal, it was considered suitable for this study and previous work has demonstrated reliability in parental self-reports regarding child 
416 feeding (36) and the CEBQ has been shown to correlate well with children's food intake (34).

417 Furthermore, the cross-sectional nature of this study means that causality cannot be inferred

418 and so future longitudinal work would help to elucidate the direction of some of the feeding

419 relationships seen in this study.

$420 \quad$ Implications for research and practice

421 Predictors of mothers' and fathers' feeding practices were examined for an

422 amalgamated sample of boys and girls and, although there were no significant differences

423 between boys' and girls' outcome variables in this study, the study's sample size was not

424 large enough for an examination of the potentially different models of feeding practices

425 dependent on child gender. However, in view of the literature which states the importance of

426 considering child gender when examining feeding interactions $(8,11,16,37)$, future work

427 should continue to examine the role of child gender in the prediction of parental feeding

428 practices.

429 The inclusion of pairs of mothers and fathers will broaden the potential implications of

430 this work for professionals working within the fields of nutrition, dietary behaviors and

431 obesity. Given that appropriate levels of control over children's feeding have been suggested

432 to link to more adaptive child feeding outcomes $(2,16,13)$, professionals can advise families

433 who have concerns about their children's eating regarding appropriate monitoring of food

434 intake and the reduction of excessively restrictive and pressuring feeding practices. Although

435 the participants in our study were a non-clinical sample, our findings suggest that, where

436 possible, professionals should attempt to establish both maternal and paternal concerns about

437 children's eating behaviors, given that both mothers and fathers are engaged in frequent

438 eating interactions with their children, and that their concerns relate to more controlling

439 practices in both parents. Furthermore, practitioners should pay attention to child

440 temperament and maternal mental health issues when assessing maternal feeding practices. 
441 Engaging and educating both parents about the importance of sensitive feeding practices may

442 add to the efficacy of prevention and intervention programs.

443 
1. Birch LL, Fisher JO, Davison KK. Learning to overeat: Maternal use of restrictive feeding practices promotes girls' eating in the absence of hunger. Am J Clin Nutr. 2003; 78: 215-20. strategies and their relationships to child eating and weight status. Obes Res. 2004; 12 : 1711-1722.

3. Birch LL, Fisher JO. Development of eating behaviors among children and adolescents. Pediatrics. 1998; 101: 539-549.

4. Blissett J, Farrow C. Predictors of maternal control of feeding at 1 and 2 years of age. Int J Obes. 2007; 31: 1520-1526.

5. Faith M, Berkowitz R, Stallings V, Kerns J, Storey M, Stunkard A. Parental feeding attitudes and styles and child body mass index: Prospective analysis of a geneenvironment interaction. Pediatrics. 2004; 114: e429-e436.

6. Stein A, Woolley H, McPherson K. Conflict between mothers with eating disorders and their infants during mealtimes. Br J Psychiatry. 1999; 175: 455-461.

7. Francis LA, Hofer SM, Birch LL. Predictors of maternal child-feeding style: Maternal and child characteristics. Appetite. 2001; 37: 231-243.

8. Tiggemann M, Lowes J. Predictors of maternal control over children's eating behavior. Appetite. 2002; 39: 1-7.

9. Field AE, Camargo Jr CA, Taylor B, Berkey CS, Roberts SB, Colditz GA. Peer, 60.

10. Farrow CV, Blissett JM. Is maternal psychopathology related to obesigenic feeding practices at 1 year? Obes Res. 2005; 13: 1999-2005. 
11. Haycraft E, Blissett J. Controlling feeding practices and psychopathology in a nonclinical sample of mothers and fathers. Eat Behav. 2008; 9: 484-493.

12. Wardle J, Guthrie CA, Sanderson S, Rapoport L. Development of the children's eating behavior questionnaire. J Child Psychol Psychiatry. 2001; 42: 963-970

13. Galloway AT, Fiorito L, Lee Y, Birch LL. Parental pressure, dietary patterns and weight status among girls who are "picky eaters". J Am Diet Assoc. 2005; 105: 541548.

14. Hagekull B, Bohlin G, Rydell AM. Maternal sensitivity, infant temperament, and the development of early feeding problems. Infant Ment Health J. 1997; 18, 92-106.

15. Pliner P, Loewen ER. Temperament and food neophobia in children and their mothers. Appetite. 1997; 28, 239-254.

16. Blissett J, Meyer C, Haycraft E. Maternal and paternal controlling feeding practices with male and female children. Appetite. 2006; 47: 212-219.

17. Haycraft E.L, Blissett J.M. Maternal and paternal controlling feeding practices: Reliability and relationships with BMI. Obesity (Silver Spring). 2008; 16: 1552-1558.

18. Johannsen DL, Johannsen NM, Specker BL. Influence of parents' eating behaviors and child feeding practices on children's weight status. Obes Res. 2006; 14: 431-439.

19. Office for National Statistics. The National Statistics Socio-Economic Classification user manual (http://www.statistics.gov.uk/methods_quality/ns_sec); 2005.

20. Birch LL, Fisher JO, Grimm-Thomas K, Markey CN, Sawyer R, Johnson SL. Confirmatory factor analysis of the Child Feeding Questionnaire: A measure of parental attitudes, beliefs and practices about child feeding and obesity proneness. Appetite. 2001; 36: 201-210.

21. Buss AH, Plomin R. Theory and measurement of EAS. In Temperament: Early developing personality traits. Hillsdale, New Jersey: Lawrence Erlbaum Associates, 1984, pp. 84-104. 
22. Mathiesen KS, Tambs K. The EAS temperament questionnaire: Factor structure, age trends, reliability and stability in a Norwegian sample. J Child Psychol Psychiatry. 1999; 40, 431-439.

23. Garner DM. Eating Disorders Inventory 2: Professional manual. Odessa, FL: Psychological Assessment Resources, 1991.

24. Spillane NS, Boerner LM, Anderson KG, Smith GT. Comparability of the Eating Disorder Inventory-2 between women and men. Assessment. 2004; 11: 85-93.

25. Derogatis LR. Brief Symptom Inventory: Administration, scoring and procedures manual. 4th edn. Minneapolis: National Computer Systems, 1993.

26. Morlan KK, Tan SY. Comparison of the Brief Psychiatric Rating Scale and the Brief Symptom Inventory. J Clin Psychol. 1998; 54: 885-94.

27. Child Growth Foundation. Cross Sectional Stature and Weight Reference Curves for the UK. London, United Kingdom: Child Growth Foundation, 1996.

28. Cole TJ, Bellizzi MC, Flegal KM, Dietz WH. Establishing a standard definition for child overweight and obesity worldwide: International survey. Br Med J. 2000; 320: 1240.

29. Taveras EM, Scanlon KS, Birch LL, Rifas-Shiman SL, Rich-Edwards JW, Gillman MW. Association of breastfeeding with maternal control of infant feeding at age 1 year. Pediatrics. 2004; 114, e577-e583.

30. Galloway AT, Fiorito LM, Francis LA, Birch LL. 'Finish your soup': Counterproductive effects of pressuring children to eat on intake and affect. Appetite. 2006; 46: 318-323.

31. Field AP. Discovering statistics using SPSS: [and sex, drugs and rock'n'roll]. 2nd edn. London: SAGE Publications, 2005.

32. Tabachnick BG, Fidell LS. Using multivariate statistics. 4th edn. Needham Heights, MA: Allyn and Bacon, 2001. 
33. Whitehouse PJ, Harris G. The inter-generational transmission of eating disorders. Eur Eat Disord Rev. 1998; 6, 238-254.

34. Carnell S, Wardle J. Measuring behavioral susceptibility to obesity: Validation of the Child Eating Behaviour Questionnaire. Appetite. 2007; 48: 104-113.

35. Saelens BE, Ernst MM, Epstein LH. Maternal child feeding practices and obesity: A discordant sibling analysis. Int J Eat Disord. 2000; 27: 459-463.

36. Whelan E, Cooper PJ. The association between childhood feeding problems and maternal eating disorder: A community study. Psychol Med. 2000; 30: 69-77.

37. Costanzo PR, Woody EZ. Domain-specific parenting styles and their impact on the child's development of particular deviance: The example of obesity proneness. $J$ Soc Clin Psychol. 1985; 3: 425-45. 
Table 1: Descriptive and reliability statistics for mothers and fathers on the Child Feeding

535 Questionnaire, Children's Eating Behaviour Questionnaire, EAS Temperament Survey,

536 Eating Disorder Inventory-2, and Brief Symptom Inventory

\begin{tabular}{|c|c|c|c|c|}
\hline & \multicolumn{2}{|c|}{ Mothers $(\mathrm{n}=48)$} & \multicolumn{2}{|c|}{ Fathers $(n=48)$} \\
\hline & Mean (SD) & $\begin{array}{c}\text { Cronbach's } \\
\text { alpha }\end{array}$ & Mean (SD) & $\begin{array}{c}\text { Cronbach's } \\
\text { alpha }\end{array}$ \\
\hline \multicolumn{5}{|c|}{ Child Feeding Questionnaire } \\
\hline Monitoring & $4.44(0.75)$ & .94 & $3.44(0.97)$ & .94 \\
\hline Pressure to Eat & $2.97(0.81)$ & .58 & $3.07(0.88)$ & .54 \\
\hline Restriction & $3.43(0.79)$ & .76 & $3.50(0.76)$ & .74 \\
\hline \multicolumn{5}{|c|}{ Child Eating Behaviour Questionnaire } \\
\hline Food Responsiveness & $2.33(0.58)$ & .74 & $2.23(0.60)$ & .69 \\
\hline Enjoyment of Food & $3.63(0.62)$ & .88 & $3.61(0.67)$ & .90 \\
\hline Satiety Responsiveness & $3.17(0.56)$ & .74 & $3.09(0.54)$ & .74 \\
\hline Food Fussiness & $3.07(0.72)$ & .88 & $2.92(0.73)$ & .90 \\
\hline Slowness in Eating & $3.05(0.63)$ & .78 & $3.06(0.64)$ & .76 \\
\hline Emotional Over-Eating & $1.77(0.55)$ & .66 & $1.81(0.52)$ & .68 \\
\hline Emotional Under-Eating & $3.26(0.77)$ & .72 & $3.27(0.76)$ & .78 \\
\hline \multicolumn{5}{|c|}{ EAS Temperament Survey } \\
\hline Shyness & $2.48(0.78)$ & .82 & $2.52(0.85)$ & .81 \\
\hline Emotionality & $2.91(0.84)$ & .88 & $2.88(0.84)$ & .84 \\
\hline Sociability $^{\dagger}$ & $4.14(0.51)$ & .60 & $3.85(0.67)$ & .74 \\
\hline Activity & $4.15(0.68)$ & .81 & $4.01(0.77)$ & .85 \\
\hline \multicolumn{5}{|c|}{ Eating Disorder Inventory-2 } \\
\hline EDI total & $16.29(12.35)$ & .94 & $7.19(6.63)$ & .80 \\
\hline \multicolumn{5}{|l|}{ Brief Symptom Inventory } \\
\hline Global Severity Index & $53.08(9.82)$ & .96 & $52.15(11.16)$ & .93 \\
\hline
\end{tabular}


542 Table 2: Two-tailed Spearman's correlations between controlling feeding practices (CFQ)

543 and children's eating behaviors (CEBQ), children's temperament (EAS), parental eating

544 disorder symptoms (EDI-2), and parental mental health (BSI) for mothers and for fathers.

\begin{tabular}{|c|c|c|c|c|c|c|}
\hline & \multicolumn{3}{|c|}{ Mothers $(n=48)$} & \multicolumn{3}{|c|}{ Fathers $(n=48)$} \\
\hline & Mon & $\mathrm{PtE}$ & Rest & Mon & $\mathrm{PtE}$ & Rest \\
\hline \multicolumn{7}{|c|}{ Child Eating Behaviour Questionnaire } \\
\hline Food Responsiveness & .012 & -.039 & .275 & -.274 & -.056 & .083 \\
\hline Enjoyment of Food & -.046 & $-.361 *$ & .001 & .059 & -.254 & .033 \\
\hline Satiety Responsiveness & -.210 & .138 & .182 & -.266 & .188 & .124 \\
\hline Food Fussiness & -.040 & $.332 *$ & .011 & -.248 & $.304 *$ & .132 \\
\hline Slowness in Eating & .068 & $.387 * *$ & .106 & -.221 & $.397 * *$ & $.287 *$ \\
\hline Emotional Over-Eating & $-.357 *$ & $.300^{*}$ & $.385 * *$ & -.217 & .049 & .091 \\
\hline Emotional Under-Eating & -.023 & $.403^{* *}$ & $.299 *$ & $-.397 * *$ & .067 & .059 \\
\hline \multicolumn{7}{|c|}{ EAS Temperament Survey } \\
\hline Shyness & .158 & .039 & .188 & -.273 & .241 & .259 \\
\hline Emotionality & -.059 & .254 & .197 & -.122 & .173 & $.316^{*}$ \\
\hline Sociability & $-.354 *$ & .042 & .011 & $.347 *$ & -.161 & -.260 \\
\hline Activity & -.153 & .085 & .129 & .228 & -.096 & -.126 \\
\hline \multicolumn{7}{|c|}{ Eating Disorder Inventory-2 } \\
\hline EDI total & -.167 & .210 & .113 & -.231 & .071 & .140 \\
\hline \multicolumn{7}{|l|}{ Brief Symptom Inventory } \\
\hline Global Severity Index & .016 & $.376^{* *}$ & .256 & -.263 & .248 & .227 \\
\hline
\end{tabular}


Table 3: Stepwise regression analyses to predict monitoring, pressure to eat and restriction in mothers $(n=48)$ and in fathers $(n=48)$

\begin{tabular}{|c|c|c|c|c|c|c|c|c|c|c|}
\hline Feeding & \multicolumn{5}{|c|}{ Maternal predictors } & \multicolumn{5}{|c|}{ Paternal predictors } \\
\hline predicted & & & & predictors & predictors & & & & predictors & predictors \\
\hline \multirow[t]{3}{*}{ Monitoring } & Sociability & 22.2 & $6.41 * *$ & $-2.77 * *$ & $-.36 * *$ & Emotional & 21.6 & $12.11 * * *$ & $-3.48 * * *$ & $-.347 * * *$ \\
\hline & Emotional Over- & & & $-2.35^{*}$ & $-.31 *$ & Under-Eating & & & & \\
\hline & Eating & & & & & & & & & \\
\hline Pressure to & Emotional & 26.5 & $8.12 * * *$ & $2.70 * *$ & $.36 * *$ & Slowness in & 19.6 & $11.24 * *$ & $3.35^{* *}$ & $.44 * *$ \\
\hline \multirow[t]{2}{*}{ Restriction } & Emotional Over- & 10.1 & $5.15^{*}$ & $2.27 *$ & $.32 *$ & Slowness in & 15.7 & $8.18^{* *}$ & $2.86^{* *}$ & $.40 * *$ \\
\hline & Eating & & & & & eating & & & & \\
\hline
\end{tabular}

